\title{
OSTEOGENIC DIFFERENTIATION OF MESENCHYMAL STEM CELLS IS REGULATED BY OSTEOCYTE AND OSTEOBLAST CELLS IN A SIMPLIFIED BONE NICHE
}

\author{
E. Birmingham ${ }^{1,2}$, G.L. Niebur ${ }^{2,3}$, P.E. McHugh $^{1,2}$, G. Shaw ${ }^{2,3}$, F.P. Barry ${ }^{2,3}$ and L.M. McNamara ${ }^{1,2 *}$
}

\begin{abstract}
${ }^{1}$ Centre for Biomechanics Research (BMEC), Mechanical and Biomedical Engineering, NUI, Galway, Ireland. ${ }^{2}$ National Centre for Biomedical Engineering Science (NCBES), NUI, Galway, Ireland. ${ }^{3}$ Department of Aerospace and Mechanical Engineering, University of Notre Dame, Notre Dame, IN 46556, USA. ${ }^{4}$ Regenerative Medicine Institute (REMEDI), NUI, Galway, Ireland.
\end{abstract}

\begin{abstract}
Mesenchymal stem cells (MSCs) within their native environment of the stem cell niche in bone receive biochemical stimuli from surrounding cells. These stimuli likely influence how MSCs differentiate to become bone precursors. The ability of MSCs to undergo osteogenic differentiation is well established in vitro; however, the role of the natural cues from bone's regulatory cells, osteocytes and osteoblasts in regulating the osteogenic differentiation of MSCs in vivo are unclear. In this study we delineate the role of biochemical signalling from osteocytes and osteoblasts, using conditioned media and co-culture experiments, to understand how they direct osteogenic differentiation of MSCs. Furthermore, the synergistic relationship between osteocytes and osteoblasts is examined by transwell co-culturing of MSCs with both simultaneously. Osteogenic differentiation of MSCs was quantified by monitoring alkaline phosphatase (ALP) activity, calcium deposition and cell number. Intracellular ALP was found to peak earlier and there was greater calcium deposition when MSCs were co-cultured with osteocytes rather than osteoblasts, suggesting that osteocytes are more influential than osteoblasts in stimulating osteogenesis in MSCs. Osteoblasts initially stimulated an increase in the number of MSCs, but ultimately regulated MSC differentiation down the same pathway. Our novel coculture system confirmed a synergistic relationship between osteocytes and osteoblasts in producing biochemical signals to stimulate the osteogenic differentiation of MSCs. This study provides important insights into the mechanisms at work within the native stem cell niche to stimulate osteogenic differentiation and outlines a possible role for the use of co-culture or conditioned media methodologies for tissue engineering applications.
\end{abstract}

Key words: Bone, mesenchymal stem cells, osteogenesis, osteoblast, osteocyte, in vitro, niche

\author{
*Address for correspondence: \\ Dr. Laoise M. McNamara \\ Department of Mechanical and Biomedical Engineering \\ National University of Ireland Galway \\ Galway, Ireland
}

Phone: (353) 91-492251

Fax: (353) 91-563991

Email: Laoise.McNamara@nuigalway.ie

\section{Introduction}

Bone marrow derived mesenchymal stem cells (MSCs) have been suggested as a suitable option for cell-based tissue engineering therapies. This is due to their capacity for self-renewal and their ability to differentiate into numerous different tissue types, such as bone, cartilage and fat (Dominici et al., 2006). While MSC strategies have proved somewhat successful for regeneration of certain tissues, such as tendons and cartilage (Butler et al., 2010; Awad et al., 2003; Haleem et al., 2010), it is not yet clear precisely how osteogenesis can be optimised. A particular limitation of current strategies is that the bone tissue produced is not adequately stiff to serve load-bearing functions in large defects in the body (Dawson and Oreffo, 2008). Therefore, the field of bone tissue regeneration is faced with the specific challenge to develop novel tissue regeneration approaches to produce large bone constructs for clinical applications.

When MSCs are cultured in osteogenic media they express markers known to be expressed by bone forming osteoblasts, which are the cells responsible for laying down the matrix and mineral during new bone formation in vivo. The osteogenic differentiation of MSCs in vitro has been divided into three stages (Huang et al., 2007). The first stage consists of days one to four where a peak in the number of cells is seen. This is followed by early cell differentiation from days 5 to 14, which is characterised by the transcription and protein expression of alkaline phosphatase (ALP) (Aubin, 2001). After this initial peak of ALP its level starts to decline. Also found at an early stage is the expression of a collagen type I matrix onto which the mineral is deposited (Quarles et al., 1992). The final stage from days 14 to 28 results in a high expression of osteocalcin and osteopontin, followed by calcium and phosphate deposition (Hoemann et al., 2009; Huang et al., 2007).

The differentiation of MSCs in vitro largely depends on the culture conditions. Growth factors, such as the TGF- $\beta$ family, result in chondrogenic differentiation (Mackay et al., 1998), while MSCs cultured with dexamethasone, insulin, isobutyl methyl xanthine, and indomethacin will differentiate down an adipogenic lineage (Pittenger et al., 1999). Osteogenic differentiation of MSCs in vitro is induced by the presence of dexamethasone, ascorbic acid and $\beta$-glycerol phosphate (Jaiswal et al., 1997). While the importance of each of these constituents for in vitro differentiation is becoming increasingly clear (Vater et al., 2011), the in vivo biochemical environment has not been well characterised and the driving source 
for the osteogenic differentiation of MSCs in their native environment remains unclear.

As biochemical and mechanical factors influencing MSC fate within their native environment are different from those used in tissue regeneration strategies and cell culture studies in vitro, it is likely that approaches for bone tissue regeneration would be enhanced if the in vivo environment was better understood. In vivo, MSCs are found within a unique environment known as the stem cell niche (Li and Xie, 2005). The stem cell niche in bone consists of a host of different support cells including haematopoietic progenitors and their progeny - such as blood cells, immune cells, and osteoclasts - and MSCs and their progeny, including fibroblasts, endothelial cells, adipocytes, osteoblasts, as well as osteocytes found embedded in bone (Kuhn and Tuan, 2010). Schofield (1978) identified the niche as having three main functions (1) maintaining quiescence, (2) promoting cell number, and (3) directing differentiation. It is likely that these support cells play a role in directing the functions of the stem cell niche (Fuchs et al., 2004). In particular it is believed that osteoblasts and osteocytes are key regulators of osteogenesis (Gu et al., 2001; Heino et al., 2004; Csaki et al., 2009), but how exactly these cells regulate the osteogenic differentiation of MSCs is not yet understood.

The aim of the present study is to examine the influence of secreted factors from osteoblasts and osteocytes on the osteogenic differentiation of MSCs. It is hypothesised that the osteogenic differentiation of MSCs in vivo is regulated by osteoblasts and osteocytes. The role of osteoblasts and osteocytes as bone regulatory cells is examined, focusing on the osteogenic response induced in MSCs when exposed to factors from osteoblast and osteocyte cells. This is examined using two different studies: (1) conditioned media and (2) indirect coculture. It is hypothesised that there exists a communication network between osteocytes and osteoblasts, which produces a stronger response in MSCs compared to either osteocytes or osteoblasts alone. This is examined using a novel three layer co-culture system, allowing physical connections between osteocytes and osteoblasts and exposing MSCs to the biochemicals produced.

\section{Materials and Methods}

\section{BALB/c MSC: isolation and characterisation}

$\mathrm{BALB} / \mathrm{c}$ MSC cultures were obtained and characterised according to the protocols of Peister et al. (2004). Briefly, 8 male mice 8 to 10 weeks old were used and cells were pooled. The femurs and tibiae were removed and cleaned of any attached tissue, and placed in complete isolation medium (CIM). CIM consisted of RPMI-1640 (Sigma Aldrich, Dublin, Ireland) supplemented with $9 \%$ foetal bovine serum (FBS, EU Thermo Scientific, Loughborough, UK), $9 \%$ horse serum (HS; Sigma Aldrich), $100 \mathrm{U} / \mathrm{mL}$ penicillin (Sigma Aldrich), $100 \mathrm{~g} / \mathrm{mL}$ streptomycin (Sigma Aldrich), and $2 \mathrm{mM}$ L-glutamine (Sigma Aldrich). The ends of the bone were clipped off and the bone was placed in an Eppendorf tube containing a P200 wide bore tip. The bones were centrifuged at $400 \mathrm{~g}$ for $2 \mathrm{~min}$ and the cell pellet was resuspended in $3 \mathrm{~mL}$ CIM. Cells were incubated in a T175 flask with $30 \mathrm{~mL}$ CIM. After $24 \mathrm{~h}$, the medium was removed and cells were washed with sterile phosphatebuffered saline (PBS, Sigma). $30 \mathrm{~mL}$ fresh CIM was added and media changed every 3 to $4 \mathrm{~d}$. After $4 \mathrm{~d}$, large colonies were observed. Cells were trypsinised and replated in CIM, and after $10 \mathrm{~d}$ cells were trypsinised again and seeded at 50 cells $/ \mathrm{cm}^{2}$ in CIM. After 3 weeks, cells were trypsinised and frozen in Iscove's modified Dulbecco medium (IMDM, Sigma Aldrich) with $5 \%$ DMSO (Sigma Aldrich) and 30 $\%$ FBS. For experiments, BALB/c MSCs were thawed and maintained in complete expansion medium (CEM) containing IMDM supplemented with $10 \%$ FBS, $10 \%$ HS, $2 \mathrm{mM}$ L-glutamine, $100 \mathrm{U} / \mathrm{mL}$ penicillin and $100 \mu \mathrm{g} /$ $\mathrm{mL}$ streptomycin until confluent.

To confirm the adipogenic potential of the MSCs used, $2 \times 10^{4}$ cells $/ \mathrm{cm}^{2}$ were incubated in CEM until they reached confluency. Then, adipogenic induction medium was added containing DMEM (Sigma Aldrich) with $10 \%$ FBS, 100 $\mathrm{U} / \mathrm{mL}$ penicillin, $100 \mathrm{~g} / \mathrm{mL}$ streptomycin, $10 \mu \mathrm{g} / \mathrm{mL}$ insulin (Sigma Aldrich), $200 \mu \mathrm{M}$ indomethacin (Sigma Aldrich), $1 \mu \mathrm{M}$ dexamethasone, and $500 \mu \mathrm{M}$ MIX (Sigma Aldrich). After $3 \mathrm{~d}$, maintenance medium was added to the cells, containing DMEM, $10 \% \mathrm{FBS}, 10 \mu \mathrm{g} / \mathrm{mL}$ insulin, $100 \mathrm{U} /$ $\mathrm{mL}$ penicillin and $100 \mathrm{~g} / \mathrm{mL}$ streptomycin. Three cycles of induction and maintenance media were completed. The cells were fixed with $10 \%$ formalin (Sigma Aldrich) and stained with $0.5 \%$ Oil Red O (Sigma Aldrich) in methanol (Sigma Aldrich).

To confirm the osteogenic potential of the MSCs used, $\mathrm{BALB} / \mathrm{c}$ MSCs $\left(2 \times 10^{4}\right.$ cells $\left./ \mathrm{cm}^{2}\right)$ were incubated in CEM until a confluent layer was achieved and then osteogenic medium was added, containing IMDM supplemented with $9 \%$ FBS, $9 \%$ HS, 2 mM L-glutamine, $100 \mathrm{U} / \mathrm{mL}$ penicillin, $100 \mu \mathrm{g} / \mathrm{mL}$ streptomycin, $50 \mathrm{ng} / \mathrm{mL} \mathrm{L}$-thyroxine (Sigma Aldrich), $20 \mathrm{mM} \beta$-glycerol phosphate, (Sigma Aldrich), $100 \mathrm{nM}$ dexamethansone (Sigma Aldrich) and 50 $\mu \mathrm{M}$ ascorbic acid (Sigma Aldrich). Medium was changed every 3-4 d. After $17 \mathrm{~d}$, cells were fixed in $10 \%$ formalin and stained with $10 \%$ Alizarin Red (Sigma Aldrich).

To confirm the chondrogenic potential of the MSCs used, $5 \times 10^{5}$ cells were seeded in pellet culture. Cells were incubated in $500 \mu \mathrm{L}$ of complete chondrogenic medium (CCM), containing IMDM, $100 \mathrm{nM}$ dexamethasone, $50 \mu \mathrm{g} / \mathrm{mL}$ ascorbic acid, $40 \mu \mathrm{g} / \mathrm{mL}$ L-proline (Sigma Aldrich), ITS+supplement (Sigma Aldrich), $1 \mathrm{mM}$ sodium pyruvate (Sigma Aldrich), $100 \mathrm{U} / \mathrm{mL}$ penicillin, $100 \mu \mathrm{g} / \mathrm{mL}$ streptomycin, $10 \mathrm{ng} / \mathrm{mL}$ TGF $\beta-3$ (Sigma Aldrich) and 100 ng/mL BMP-2 (Sigma Aldrich). The medium was changed three times a week. After $21 \mathrm{~d}$, pellets were harvested, embedded in paraffin and stained with Toluidine Blue sodium borate (Sigma Aldrich) and Safranin O (Sigma Aldrich).

\section{MLO-Y4 and MC3T3-E1 cell cultures}

Two bone cell lines were used in this study. MLO-Y4 cells are a murine-derived cell line, which shares numerous characteristics with primary osteocytes, such as high production of osteocalcin and low expression of alkaline phosphatase as well as producing numerous dendritic processes (Kato et al., 1997). MLO-Y4 cell cultures were 
maintained on collagen coated plates in $\alpha$-modified minimal essential medium ( $\alpha$-MEM) supplemented with $2.5 \%$ FBS, $2.5 \%$ iron supplemented calf serum (CS, HyClone Laboratories, Logan, UT, USA), 2 mM L-glutamine, 100 $\mathrm{U} / \mathrm{mL}$ penicillin and $100 \mu \mathrm{g} / \mathrm{mL}$ streptomycin. MC3T3-E1 cells are an osteoblast cell line, which express high amounts of alkaline phosphatase, produce mineral and are capable of differentiating into osteocytes, making them appropriate for osteoblast studies (Sudo et al., 1983). They are considered to be a good model of primary osteoblasts when treated with osteogenic media (Quarles et al., 1992). The MC3T3-E1 cells were maintained in $\alpha$-MEM supplemented with $5 \%$ FBS, $2 \mathrm{mM}$ L-glutamine, $100 \mathrm{U} / \mathrm{mL}$ penicillin and $100 \mu \mathrm{g} / \mathrm{mL}$ streptomycin.

\section{Conditioned media (CM) experiment}

MC3T3-E1 cells were plated at a density of 5,200 cells $/ \mathrm{cm}^{2}$ and were pre-treated for $24 \mathrm{~h}$ in osteogenic medium ( $\alpha \mathrm{MEM}$, $10 \%$ FBS, $2 \mathrm{mM}$ L-glutamine, $100 \mathrm{U} / \mathrm{mL}$ penicillin, 100 $\mu \mathrm{g} / \mathrm{mL}$ streptomycin, $20 \mathrm{mM} \beta$-glycerol phosphate, 50 $\mu \mathrm{M}$ ascorbic acid and $100 \mathrm{nM}$ dexamethasone). This pretreatment was carried out to establish the osteoblast cell phenotype prior to the beginning of the study (Kato et al., 1997; Sudo et al., 1983). After pretreatment, cells were cultured in standard osteoblast media ( $\alpha$-MEM, $5 \%$ FBS, $2 \mathrm{mM}$ L-glutamine, $100 \mathrm{U} / \mathrm{mL}$ penicillin and $100 \mu \mathrm{g} /$ $\mathrm{mL}$ streptomycin) for $24 \mathrm{~h}$. MLO-Y4 cells were plated in separate 6 well plates at the same density in their standard osteocyte medium ( $\alpha$-MEM with $2.5 \%$ FBS, $2.5 \% \mathrm{CS}$, $2 \mathrm{mM}$ L-glutamine, $100 \mathrm{U} / \mathrm{mL}$ penicillin and $100 \mu \mathrm{g} / \mathrm{mL}$ streptomycin) for $24 \mathrm{~h}$. MSC medim was supplemented with conditioned media collected from either the MLO-Y4 cells (MLO-Y4 CM) or the MC3T3-E1 cells (MC3T3-E1 $\mathrm{CM}$ ), centrifuged at $300 \mathrm{~g}$, and the supernatant added at a ratio of 2:1 to MSCs (yielding a $33 \%$ dilution), which had been plated at a density of 5,200 cells $/ \mathrm{cm}^{2}$ in separate 6 well plates for $24 \mathrm{~h}$. Medium was changed every $3 \mathrm{~d}$, including fresh conditioned medium each time. Controls consisted of MSCs cultured in CEM and a positive control of MSCs with osteogenic medium (IMDM with $10 \% \mathrm{FBS}$, $10 \% \mathrm{HS}, 2 \mathrm{mM}$ L-glutamine, $100 \mathrm{U} / \mathrm{mL}$ penicillin, 100 $\mu \mathrm{g} / \mathrm{mL}$ streptomycin, $20 \mathrm{mM} \beta$-glycerol phosphate, 50 $\mu \mathrm{M}$ ascorbic acid and $100 \mathrm{nM}$ dexamethasone). As for all experiments, MSCs osteogenic differentiation of MSCs was quantified by monitoring alkaline phosphatase (ALP) activity, calcium deposition and cell number at 2, 3, 7, 10 and $14 \mathrm{~d}$, as described in detail below.

\section{Co-culture (CC) experiment}

MSCs were simultaneously cultured with either osteoblasts or osteocytes using inserts containing permeable membranes (PET $1 \mu \mathrm{m}$ pores; Millipore, Cork, Ireland). These membranes ensured that osteoblasts or osteocytes were physically separated from MSCs by a distance of 0.9 $\mathrm{mm}$ to prevent direct cell-to-cell contact. MSCs $(5,200$ cells $/ \mathrm{cm}^{2}$ ) were cultured for $24 \mathrm{~h}$ on the bottom of 6 well plates in appropriate media and then exposed to either a MLO-Y4 (MLO-Y4 CC), MC3T3-E1 (MC3T3-E1 CC) or a three layer co-culture (3CC) seeded insert. MSCs were also exposed to a control of an MSC-seeded membrane (MSC CC). For the MC3T3-E1 CC group, MC3T3-E1 cells were seeded at 5,200 cells $/ \mathrm{cm}^{2}$ on the apical side of the insert membranes and pre-treated for $24 \mathrm{~h}$ in osteogenic medium before being cultured in standard osteoblast medium for $24 \mathrm{~h}$. For the MLO-Y4 CC group, MLO-Y4 cells were seeded at the same density on the apical side of the insert membranes with their standard osteocyte medium. The three-layered co-culture (3CC) was prepared with MLO-Y4 cells seeded on the apical side of the insert membrane and MC3T3-E1 cells on the basal side of the same membrane. The pore size was chosen to allow the MLO-Y4 and MC3T3-E1 to form functional gap junctions across the membrane (Taylor et al., 2007). To achieve this, inserts were first turned upside down and 2,600 cells/ $\mathrm{cm}^{2} \mathrm{MC} 3 \mathrm{~T} 3-\mathrm{E} 1$ cells were seeded on the basal surface in $500 \mu \mathrm{L}$ medium. These cells were allowed to attach for $6 \mathrm{~h}$ before the insert was turned and seeded with 2,600 cells $/ \mathrm{cm}^{2}$ MLO-Y4 cells on the apical side. Finally, for the control MSC CC, 5,200 cells $/ \mathrm{cm}^{2}$ MSCs were seeded on the apical side of the insert membranes in CEM for $24 \mathrm{~h}$. For all co-culture studies, cells on each side of the membrane received CEM (i.e. non-osteogenic medium). MSCs were examined and quantified at 2, 3, 7, 10 and $14 \mathrm{~d}$ for cell number, ALP activity and matrix mineralisation, as described in detail below. For all the examinations outlined below, two independent experiments were conducted with at least two repeats in each experiment (total $n>4$ ).

\section{Alkaline phosphatase (ALP)}

After exposure to conditioned medium or co-culture for periods of 2, 3 and $7 \mathrm{~d}$, cells were washed with PBS and fixed with $10 \%$ formalin, and washed again three times with PBS. Extracellular ALP activity was examined histochemically using Fast Violet B salt (Sigma) and Naphthol AS-MX phosphate (Sigma). After ALP staining, the samples were washed with PBS and stained with 4',6-diamidino-2-phenylindole (DAPI, Sigma). Five images were taken at random locations in each sample for ALP staining and DAPI staining using an Olympus IX71 Inverted Fluorescent Microscope with Olympus Cell P Software. Images were analysed using Image $\mathrm{J}$ software (NIH) for cell count per $\mathrm{cm}^{2}$ (DAPI) and percentage ALP stain per total image area. Relative cell number was calculated by dividing the total cell count at day 7 by the total cell count at day 0 .

Intracellular ALP activity was measured colorimetrically using an Alkaline Phosphatase Colorimetric Assay Kit (Abcam, Cambridge, UK) which uses p-nitrophenyl phosphate (pNPP) as a phosphatase substrate. Cell lysate was prepared using three cycles of freeze-thaw in deionised distilled water. $30 \mu \mathrm{L}$ of the cell lysate was added to a 96 well plate with $50 \mu \mathrm{L}$ assay buffer and 50 $\mu \mathrm{L}$ pNPP. The samples were shielded from direct light at room temperature for $1 \mathrm{~h}$. After this, $20 \mu \mathrm{L}$ Stop Solution $(3 \mathrm{~N} \mathrm{NaOH})$ was added to the wells and the plate was read at $405 \mathrm{~nm}$ in a micro-plate reader (Wallac Victor3 1420 Multilabel Counter).

\section{DNA content}

The cell lysate (prepared for ALP assay) was measured for DNA content using the fluorescent dye bisBenzimide $\mathrm{H}$ 33258 (Hoechst 33258, Sigma). Briefly, $50 \mu \mathrm{L}$ of the cell 
lysate was added to a 96 well plate with $50 \mu \mathrm{L}$ assay buffer and $100 \mu \mathrm{L}$ Hoechst Dye. A $0.1 \mu \mathrm{g} / \mathrm{mL}$ solution was used for 2 and $3 \mathrm{~d}$ time points and a $1 \mu \mathrm{g} / \mathrm{mL}$ solution for the later time points. The plate was incubated away from light for $10 \mathrm{~min}$ and then read on a microplate reader (Wallac Victor3 1420 Multilabel Counter) at excitation of $350 \mathrm{~nm}$ and emission of $450 \mathrm{~nm}$. Cell number was determined by using a known amount of purified calf thymus DNA as the standard.

\section{Mineralisation}

Medium was removed from MSCs and the cells washed three times with PBS. They were then evaluated for calcium production at 10 and $14 \mathrm{~d}$ by staining with $10 \%$ Alizarin Red solution. Alizarin Red S is a dye that binds to calcium salts. After fixation and staining cells were again washed three times with PBS. To quantify the staining, $1 \mathrm{~mL}$ of $10 \%$ cetylpyridium chloride (Sigma) was added to each well and incubated for $20 \mathrm{~min}$ to elute the stain. $100 \mu \mathrm{L}$ of this eluted stain was added to 96 well plates and read at $550 \mathrm{~nm}$ using a spectrophotometer (Nørgaard et al., 2006). A standard curve was prepared using Alizarin Red stain and cetylpyridium chloride. The calcium deposition was expressed as molar equivalent of calcium. One mole of Alizarin Red binds to two moles of calcium in an Alizarin Red S-calcium complex.

\section{Statistical analysis}

DNA content using Hoechst staining method, increase in cell number using DAPI, extracellular ALP stained per well, in addition to intracellular ALP and extracellular calcium deposition both normalised to DNA content, were compared between groups for conditioned media and co-culture studies separately with a one-way analysis of variance (ANOVA) followed by pair-wise comparison (Tukey's HSD test). Student's $t$-test was applied to compare the calcium deposition normalised to DNA content between MLO-Y4 CC and MLO-Y4 CM groups. All analyses were performed with Minitab. For all comparisons, the level of significance was $p \leq 0.05$.

\section{Results}

The first section demonstrates the multipotent nature of the $\mathrm{BALB} / \mathrm{c}$ MSCs used in the experiments. The subsequent sections each address a specific question related to the two hypotheses presented above. Firstly, "The osteogenesis of MSCs in vivo is regulated by osteoblasts and osteocytes", and secondly, "There exists a communication network between osteocytes and osteoblast which produces a robust osteogenic response in MSCs".

\section{Characterisation of BALB/c MSCs}

Differentiation assays confirmed the multipotency of the isolated MSCs. They displayed positive markers of osteogenic, adipogenic and chondrogenic differentiation (Fig. 1).

\section{Are osteocytes the regulatory cells that govern osteogenic differentiation of MSCs?}

\section{Cell number}

MSCs that had been treated with conditioned media from MLO-Y4 cells (MLO-Y4 CM) had a lower DNA content than MSCs in their normal media (Control) at all time points, but were statistically significantly different at 2 $\mathrm{d}(p=0.0075)$ and $14 \mathrm{~d}(p=0.0179)$ (Fig. 2a). A similar trend was found for MSCs that were exposed to an insert containing MLOY4 cells (MLO-Y4 CC) with a lower DNA content, compared to the control of MSCs exposed to an MSC-seeded insert (MSC CC), at $2 \mathrm{~d}(p<0.0001), 10 \mathrm{~d}$ $(p=0.0056)$ and $14 \mathrm{~d}(p<0.0001)$ (Fig. 2b). When the relative change in cell number between day 0 and day 7 was examined, it was found that the MLO-Y4 CM group had a lower cell number than the osteogenic group $(p=0.0013)$, and the MLO-Y4 CC group was significantly lower than the MSC CC group $(p<0.0001)$ (Fig. 3).

\section{ALP activity}

The extracellular ALP activity by MSCs in the MLO-Y4 CM group was not significantly different from the control group at any time point and was lower than the osteogenic group at $7 \mathrm{~d}(p<0.0001)$ (Fig. 4a). ALP activity by MSCs in the MLO-Y4 CC group was greater than that of the MSC CC group at $7 \mathrm{~d}(p=0.0086)$ (Fig. 4b). Intracellular ALP activity in the MLO-Y4 CM group peaked early and dissipated by 7 d (Fig. 5a). At 2 d, ALP activity was greater in the MLO-Y4 CM group than control $(p<0.0001)$ and the osteogenic group $(p<0.0001)$. Although a rise in ALP activity was noted at $3 \mathrm{~d}$ compared to controls, this was not statistically different, but by $7 \mathrm{~d}$ the ALP activity in the MLO-Y4 CM group was lower than the control $(p<$ $0.0001)$ and the osteogenic group $(p=0.0005)$. An early peak was also found with the MLO-Y4 CC group in the co-culture experiments (Fig. 5b). ALP intracellular activity was greater in the MLO-Y4 CC group than the MSC CC group at $2 \mathrm{~d}(p<0.0001)$, but had decreased to control levels by day 7 .

\section{Calcium deposition}

Calcium deposition in the MLO-Y4 CM group was lower than the osteogenic group at 10 and $14 \mathrm{~d}$ (significant at 14 $\mathrm{d}, p=0.0003$ ) and had no significant difference from the control group (Fig. 6a). However calcium deposition by MSCs in the MLO-Y4 CC group was higher at $14 \mathrm{~d}$ than the MSC CC control group ( $p=0.05)$ (Fig. 6b).

\section{Are osteoblasts the regulatory cells that govern osteogenic differentiation of MSCs?}

\section{Cell number}

MSCs treated with conditioned media from MC3T3-E1 cells (MC3T3-E1 CM) had a higher DNA content than the control (MSCs in regular media) at $3 \mathrm{~d}(p=0.0003)$ but were significantly lower than control by $14 \mathrm{~d}(p<0.0001)$ (Fig. 2a). Moreover the MC3T3-E1 CM group had a higher 

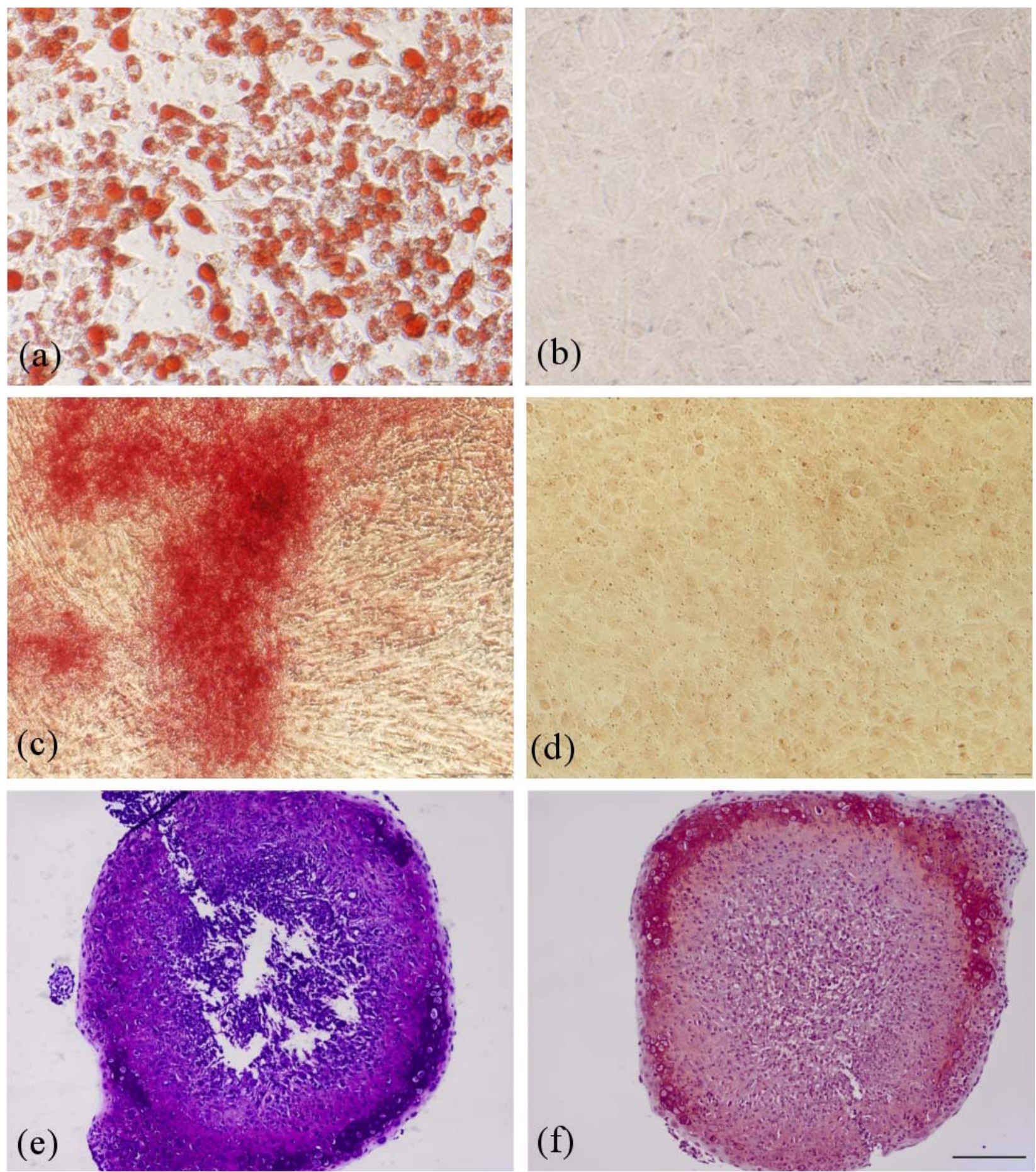

Fig. 1. Characterisation of BALB/c MSCs. Fat globules are visible in MSC adipogenic test culture (a) but not in control (b) after staining with Oil-Red-O indicating that MSCs are undergoing adipogenic differentiation. Calcium deposition can be seen after staining with Alizarin Red in the osteogenic test culture (c) showing the positive result for osteogenic differentiation compared to the control (d). Staining with Toluidine Blue for chondrocyte-associated proteoglycan in the chondrogenic test cultures was positive within the pellet as indicated by the purple colour (e). This was confirmed by Safranin O staining positive for the presence of cartilage within the pellet culture (f). Scale bar $=200 \mu \mathrm{m}$, same scale for all images. 

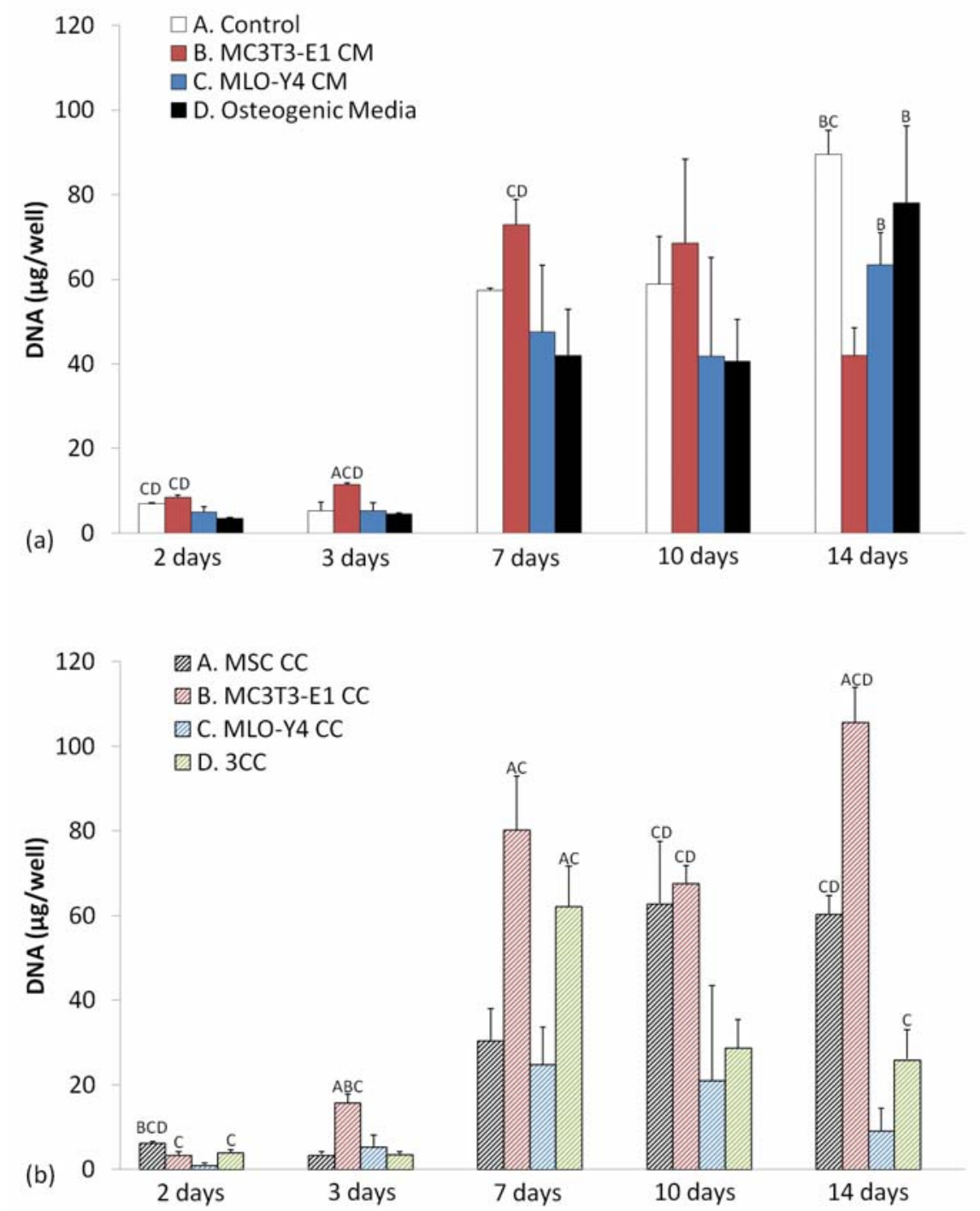

Fig. 2. DNA content at 2, 3, 7, 10 and $14 \mathrm{~d}$ as measured by the Hoechst Assay for (a) MSCs exposed to conditioned media from MLO-Y4 (MLO-Y4 CM) and MC3T3-E1 cells (MC3T3-E1 CM). Also shown is the cell DNA content for MSCs grown in normal complete expansion media (Control) and MSCs grown in osteogenic media (Osteogenic Media). A, $p<0.05$ versus Control; $\mathrm{B}, p<0.05$ versus MC3T3-E1 CM; C, $p<0.05$ versus MLO-Y4 CM; D, $p<0.05$ versus Osteogenic Media. (b) DNA content for MSCs co-cultured with MLO-Y4 cells (MLO-Y4 CC), MC3T3-E1 cells (MC3T3-E1 CC), a three-layer co-culture with both MLO-Y4 and MC3T3-E1 cells (3CC), and MSCs cocultured with MSCs (MSC CC). A, $p<0.05$ versus MSC CC; $\mathrm{B}, p<0.05$ versus MC3T3-E1 CC; $\mathrm{C}, p<0.05$ versus MLO-Y4 CC; D, $p<0.05$ versus 3CC. Error bars denote standard deviation $(n=4)$.

DNA content than the osteogenic group at all time points (2 $\mathrm{d}, p<0.0001 ; 3 \mathrm{~d}, p=0.0001 ; 7 \mathrm{~d}, p=0.0055)$. Similarly MSCs exposed to an insert containing MC3T3-E1 cells (MC3T3-E1 CC) had a high DNA content compared to the MSC CC control group ( $p=0.0001$ at $2 \mathrm{~d}$ and $p<0.0001$ at 3, 7 and 14 d) (Fig. 2b). Indeed, when the relative change in cell number was examined, the MC3T3-E1 CM group was significantly higher than the control group $(p=0.0001)$ and the osteogenic media group $(p=0.0415)$ (Fig. 3).

\section{ALP activity}

Extracellular ALP activity for the MC3T3-E1 CM group at 2 and $3 \mathrm{~d}$ was not significantly different from the controls, but was higher than the control (MSCs in normal medium) at $7 \mathrm{~d}(p=0.0495)$. However, it was significantly lower than the osteogenic group at $7 \mathrm{~d}(p=0.0001)$ (Fig. 4a). In the same way, the ALP activity for the MC3T3-E1 CC group was higher than the MSC CC group at $3 \mathrm{~d}(p<0.0001)$ and $7 \mathrm{~d}(p=0.0495)$ (Fig. 4b). Intracellular ALP activity in the MC3T3-E1 CC group was found to be lower than the osteogenic group at 2 and $3 \mathrm{~d}$ (not significant), however by $7 \mathrm{~d}$ it had the highest activity compared to the control $(p=0.0044)$ and the osteogenic group $(p=0.0001)$ (Fig. $5 \mathrm{a})$. This trend is replicated in the MC3T3-E1 CC group, as at $7 \mathrm{~d}$ it had the highest ALP activity compared to the MSC CC group ( $p=0.0003)$ (Fig. 5b).

\section{Calcium deposition}

Calcium deposition in the MC3T3-E1 CM group was similar to the control of MSCs in CEM, but was 


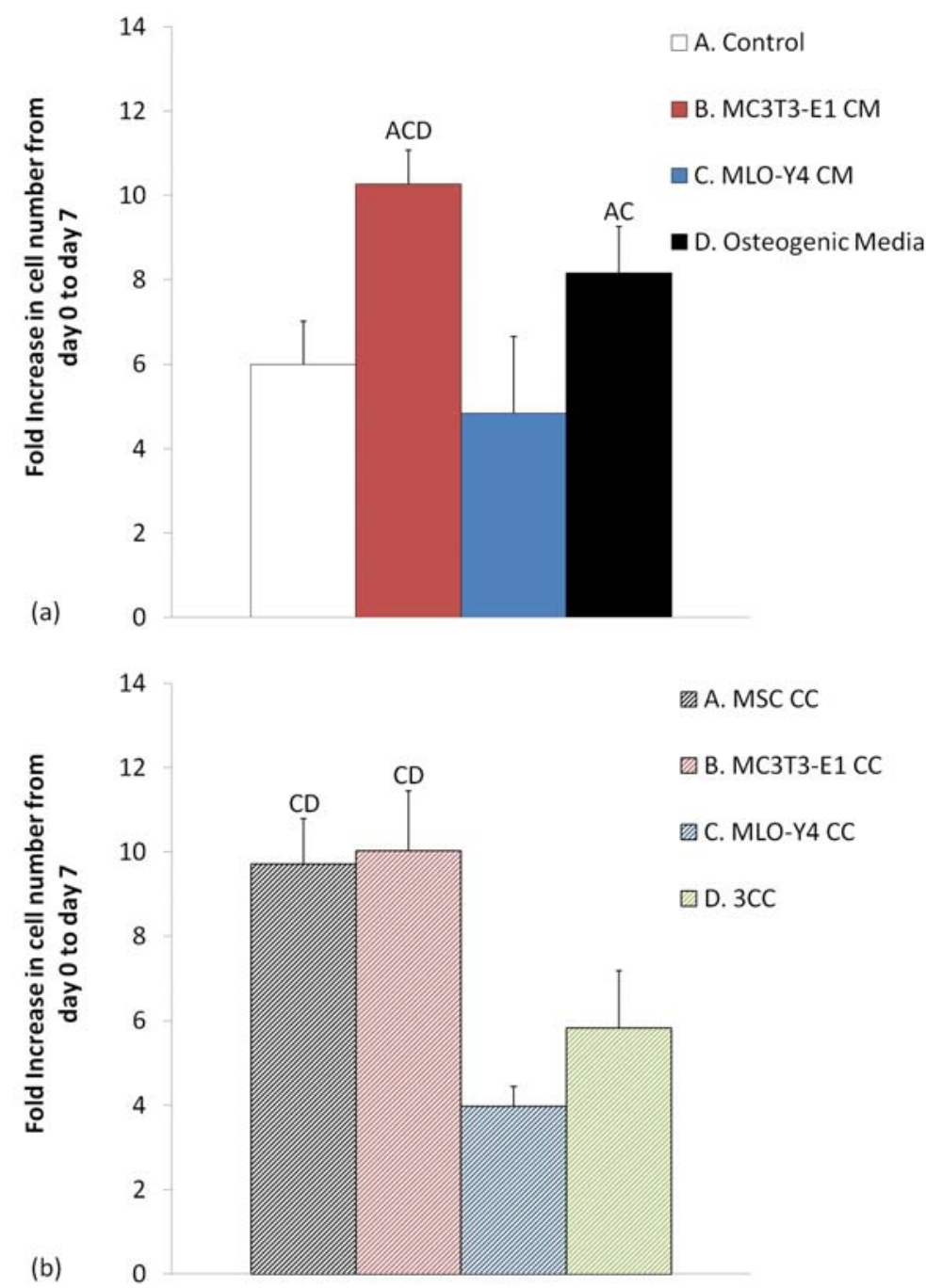

Fig. 3. Relative cell number increase from day zero to day seven (fold change) as measured by DAPI staining for MSCs (a) treated with conditioned media from MLO-Y4 cells (MLO-Y4 CM) and MC3T3-E1 cells (MC3T3-E1 $\mathrm{CM}$ ), or (b) co-cultured with MLO-Y4 cells (MLO-Y4 CC) and MC3T3-E1 cells (MC3T3-E1 CC). Results for MSCs grown in complete expansion medium (Control), MSCs grown in osteogenic media (Osteogenic Media), three-layer co-culture with both MLO-Y4 and MC3T3-E1 cells (3CC), and MSCs co-cultured with MSCs (MSC $\mathrm{CC})$ are also shown. A, $p<0.05$ versus Osteogenic Media; $\mathrm{B}, p<0.05$ versus Control; $\mathrm{C}, p<0.05$ versus MSC $\mathrm{CC} ; \mathrm{D}, p<0.05$ versus MC3T3-E1 CM; E, $p<0.05$ versus MC3T3-E1 CC; F, $p<0.05$ versus MLO-Y4 CM; G, $p$ $<0.05$ versus MLO-Y4 CC; H, $p<0.05$ versus 3CC. Relative cell number was calculated by dividing the total cell count at day 7 by the total cell count at day 0 . Error bars denote standard deviation $(n=5)$.

significantly lower than the osteogenic group at $14 \mathrm{~d}(p=$ 0.0003). Similarly, calcium deposition for the MC3T3-E1 CC group was not different from the control MSC CC group at either 10 or $14 \mathrm{~d}$ (Fig. 6).

Does the degree of osteogenic differentiation of MSCs differ between osteocyte and osteoblast signalling?

\section{Cell number}

The DNA content in the MC3T3-E1 CM group was greater than in the MLO-Y4 CM group at $2 \mathrm{~d}(p=0.0001), 3 \mathrm{~d}(p$ $=0.0003)$ and $7 \mathrm{~d}(p=0.0209)$, while at $14 \mathrm{~d}$ the order had reversed with MLO-Y4 CM group having the greater DNA content $(p<0.0001)$ (Fig. 2a). Similarly, comparing the MC3T3-E1 CC group with the MLO-Y4 CC group it can be seen that the DNA content is greater in the MC3T3-E1 CC group ( $2 \mathrm{~d}, p=0.0005 ; 3 \mathrm{~d}, p<0.0001 ; 7 \mathrm{~d}, p<0.0001$ and $14 \mathrm{~d}, p<0.0001$ ) (Fig.2b). This trend was confirmed when the relative change in cell number between day 0 and day 7 was examined; the MC3T3-E1 CM group showed a higher cell number than the MLO-Y4 CM group $(p<0.0001)$. Similarly, the MC3T3-E1 CC group showed a higher cell number than the MLOY4 CC group $(p<0.0001)$ (Fig. 3).

\section{ALP activity}

When the ALP extracellular activity was examined, no significant difference was found between MC3T3-E1 CM and MLO-Y4 CM groups (Fig. 4a), and the only significant difference between the two co-culture groups was seen at $3 \mathrm{~d}$ with MC3T3-E1 CC groups showing greater ALP 

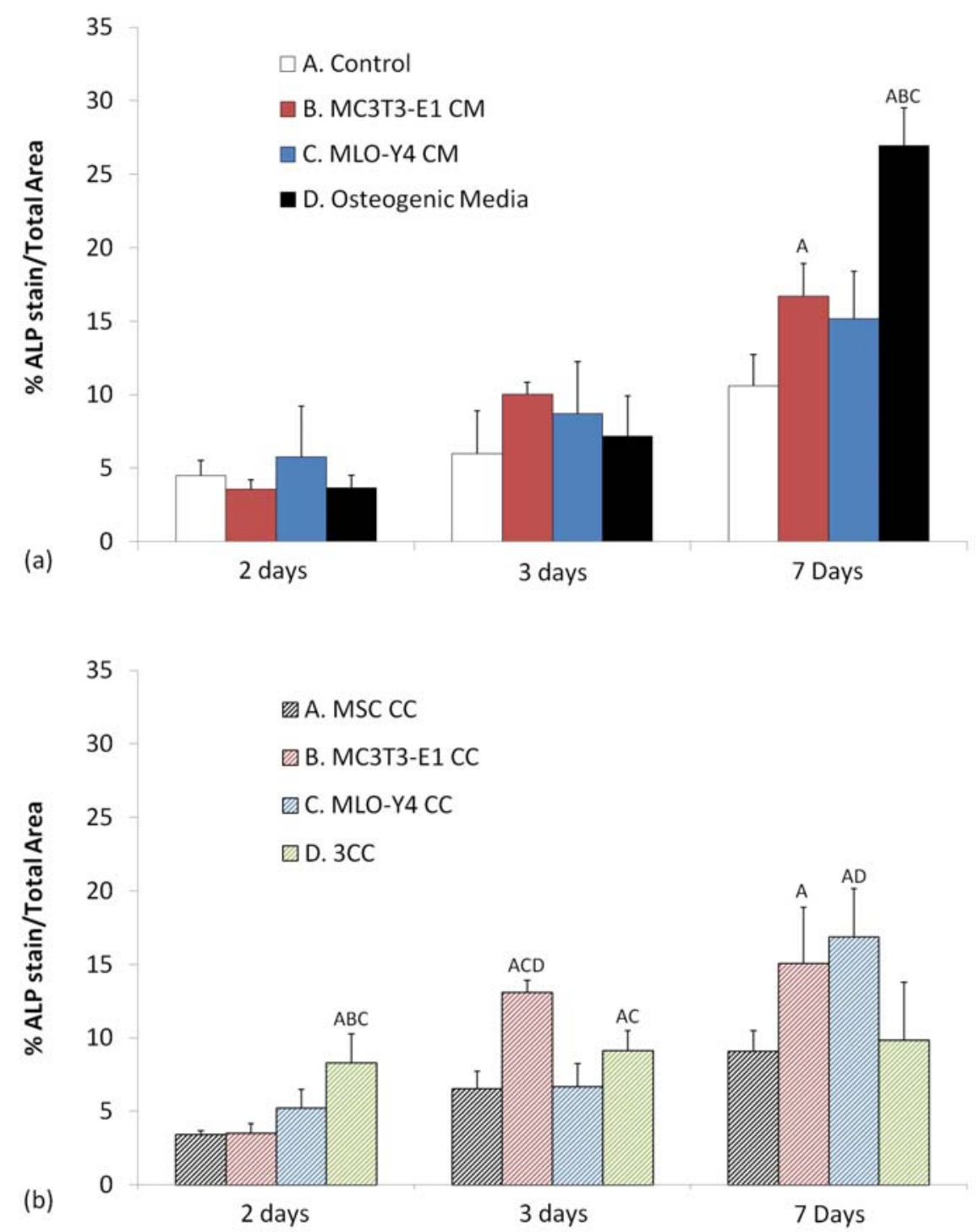

Fig. 4. Extracellular ALP activity as measured histochemically at 2, 3 and $7 \mathrm{~d}$, for (a) MSCs exposed to conditioned media from MLO-Y4 cells (MLO-Y4 CM) and MC3T3-E1 cells (MC3T3-E1 CM). Also shown is the ALP activity for MSCs grown in normal complete expansion media (Control) and MSCs grown in osteogenic media (Osteogenic Media). A, $p<0.05$ versus Control; B, $p<0.05$ versus MC3T3-E1 CM; C, $p<0.05$ versus MLO-Y4 CM; D, $p<0.05$ versus Osteogenic Media. (b) MSCs co-cultured with MLOY4 cells (MLO-Y4 CC), MC3T3-E1 cells (MC3T3-E1 $\mathrm{CC})$, a three-layer co-culture with both MLO-Y4 and MC3T3-E1 cells (3CC), and MSCs co-cultured with MSCs (MSC CC). A, $p<0.05$ versus MSC CC; $\mathrm{B}, p<0.05$ versus MC3T3-E1 CC; $\mathrm{C}, p<0.05$ versus MLO-Y4 CC; D, $p<$ 0.05 versus 3 CC. Error bars denote standard deviation $(n=5)$.

activity than the MLO-Y4 CC group $(p<0.0001)$ (Fig. $4 b)$. Examining intracellular ALP activity, it was found that at 2 and $3 \mathrm{~d}$ the ALP activity was greater in the MLO-Y4 CM group compared to the MC3T3-E1 CM group ( $p<$ 0.0001 and 0.0423 , respectively) (Fig. 5a). However, by $7 \mathrm{~d}$ the peak in the MLO-Y4 CM group had ended and the MC3T3-E1 CM group was greater $(p=0.0001)$. A similar trend was found when the MLO-Y4 CC group was compared to the MC3T3-E1 CC group, with the MLO-Y4 $\mathrm{CC}$ group having the greater ALP activity compared to the MC3T3-E1 CC group at $2 \mathrm{~d}(p=0.0004)$, while the opposite was true at $7 \mathrm{~d}(p=0.0001)$ (Fig. 5b).

\section{Calcium deposition}

There was no difference between the MLO-Y4 CM and MC3T3-E1 CM groups at either 10 or $14 \mathrm{~d}$ (Fig. 6a). However, the MLO-Y4 CC group had a greater calcium deposition at 10 and $14 \mathrm{~d}$ compared to the MC3T3-E1 CC group ( $p=0.0496$ and 0.0083 , respectively).

\section{Do osteoblasts and osteocytes work in conjunction to regulate MSC differentiation?}

\section{Cell number}

The DNA content of the MSCs exposed to both MLO-Y4 and MC3T3-E1 cells simultaneously (3CC) was higher 

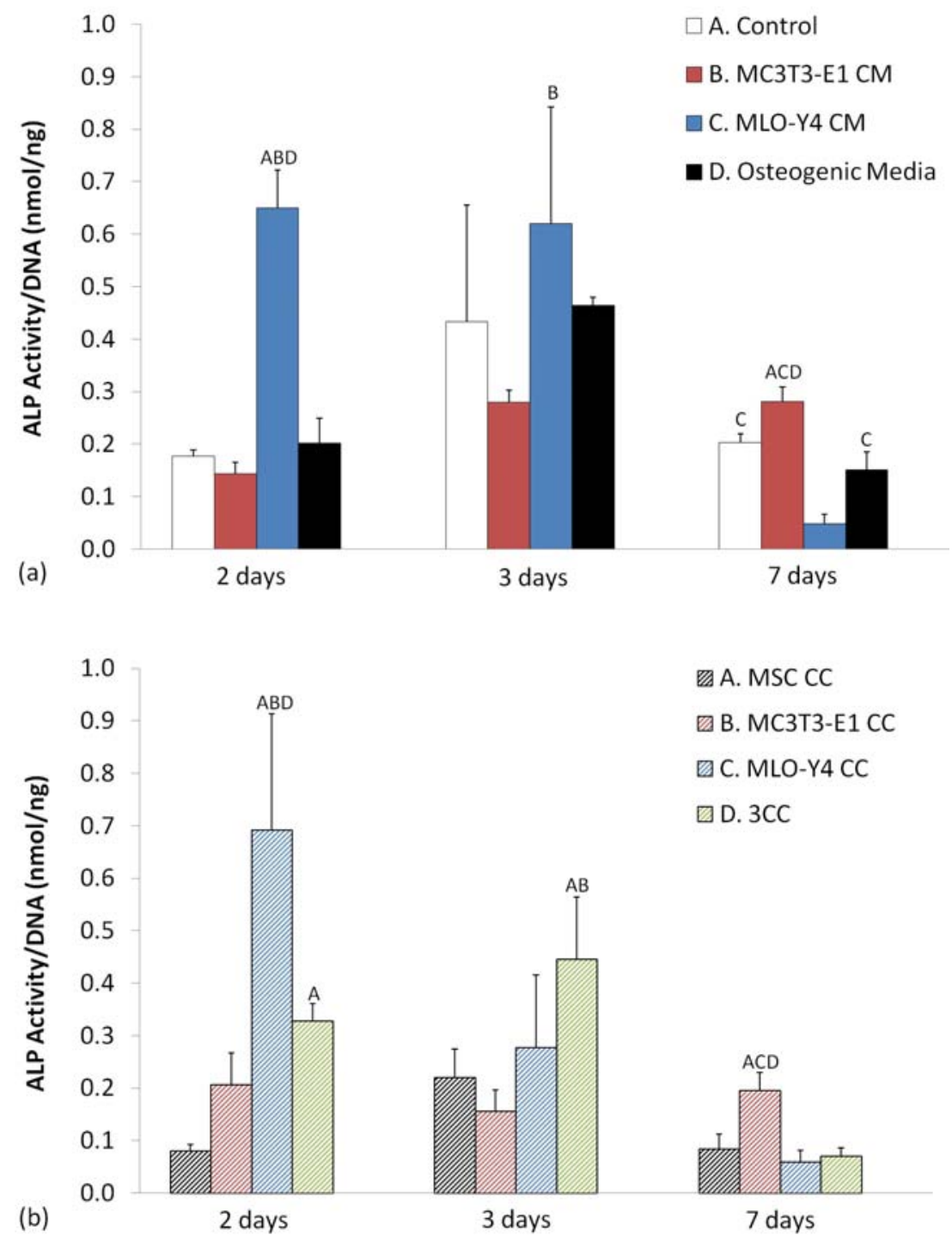

Fig. 5. Intracellular ALP activity normalised to DNA content at 2, 3 and $7 \mathrm{~d}$ for (a) MSCs exposed to conditioned media from MLO-Y4 cells (MLO-Y4 CM) and MC3T3-E1 cells (MC3T3-E1 CM). Also shown is the ALP activity for MSCs grown in normal complete expansion media (Control) and MSCs grown in osteogenic media (Osteogenic Media). A, $p<0.05$ versus Control; $\mathrm{B}, p<0.05$ versus MC3T3-E1 CM; C, $p<0.05$ versus MLO-Y4 CM; D, $p<0.05$ versus Osteogenic Media. (b) MSCs co-cultured with MLOY4 cells (MLO-Y4 CC), MC3T3-E1 cells (MC3T3-E1 $\mathrm{CC}$ ), a three-layer co-culture with both MLO-Y4 and MC3T3-E1 cells (3CC), and MSCs co-cultured with MSCs (MSC CC). A, $p<0.05$ versus MSC CC; B, $p<0.05$ versus MC3T3-E1 CC; C, $p<0.05$ versus MLO-Y4 CC; D, $p$ $<0.05$ versus 3 CC. Error bars denote standard deviation $(n=4)$.

than the MLO-Y4 CC group at $2 \mathrm{~d}(p=0.0001), 7 \mathrm{~d}(p=$ $0.0009)$ and $14 \mathrm{~d}(p=0.0139)$, while it was significantly lower than the MC3T3-E1 CC group at $3 \mathrm{~d}(p<0.0001)$, $10 \mathrm{~d}(p=0.0094)$ and $14 \mathrm{~d}(p<0.0001)$ (Fig. 2b). It was also lower than the MSC CC group at $2 \mathrm{~d}(p=0.0007)$, $7 \mathrm{~d}(p=.0032), 10 \mathrm{~d}(p=0.0220)$ and $14 \mathrm{~d}(p<0.0001)$. This is reflected when the relative change in cell number is analysed between day 0 and day 7 . It can be seen that the $3 \mathrm{CC}$ group has a lower cell number than the MC3T3-E1 CC group $(p=0.0002)$ and the MSC CC group $(p=0.0004)$ (Fig. 3).

\section{ALP activity}

The extracellular ALP activity of the $3 \mathrm{CC}$ group was greater than all the other groups at $2 \mathrm{~d}$ (MC3T3-E1 CC and
MSC CC $p=0.0001$, MLO-Y4 CC $p=0.0062)$. By $7 \mathrm{~d}$ the activity had decreased and was lower than the MLO-Y4 CC group ( $p=0.0181$ ) (Fig. 4b). For the intracellular ALP activity, it can be seen that the $3 \mathrm{CC}$ has significantly less ALP than the MLO-Y4 CC group $(p=0.0038)$ at $2 \mathrm{~d}$ but more than the MSC CC group at $2 \mathrm{~d}(p=0.0462)$ and 3 $\mathrm{d}(p=0.0337)$ and the MC3T3-E1 CC group at $3 \mathrm{~d}(p=$ 0.0090). However, by $7 \mathrm{~d}$ the ALP activity had decreased and there was less ALP activity than in the MC3T3-E1 CC group $(p=0.0001)$ (Fig. 5b).

Calcium deposition

At $10 \mathrm{~d}$ the $3 \mathrm{CC}$ group had the significantly greatest calcium deposition than all groups (MLO-Y4 CC $p=$ 0.0136, MC3T3-E1 CC $p=0.0001$, and MSC CC $p=$ 

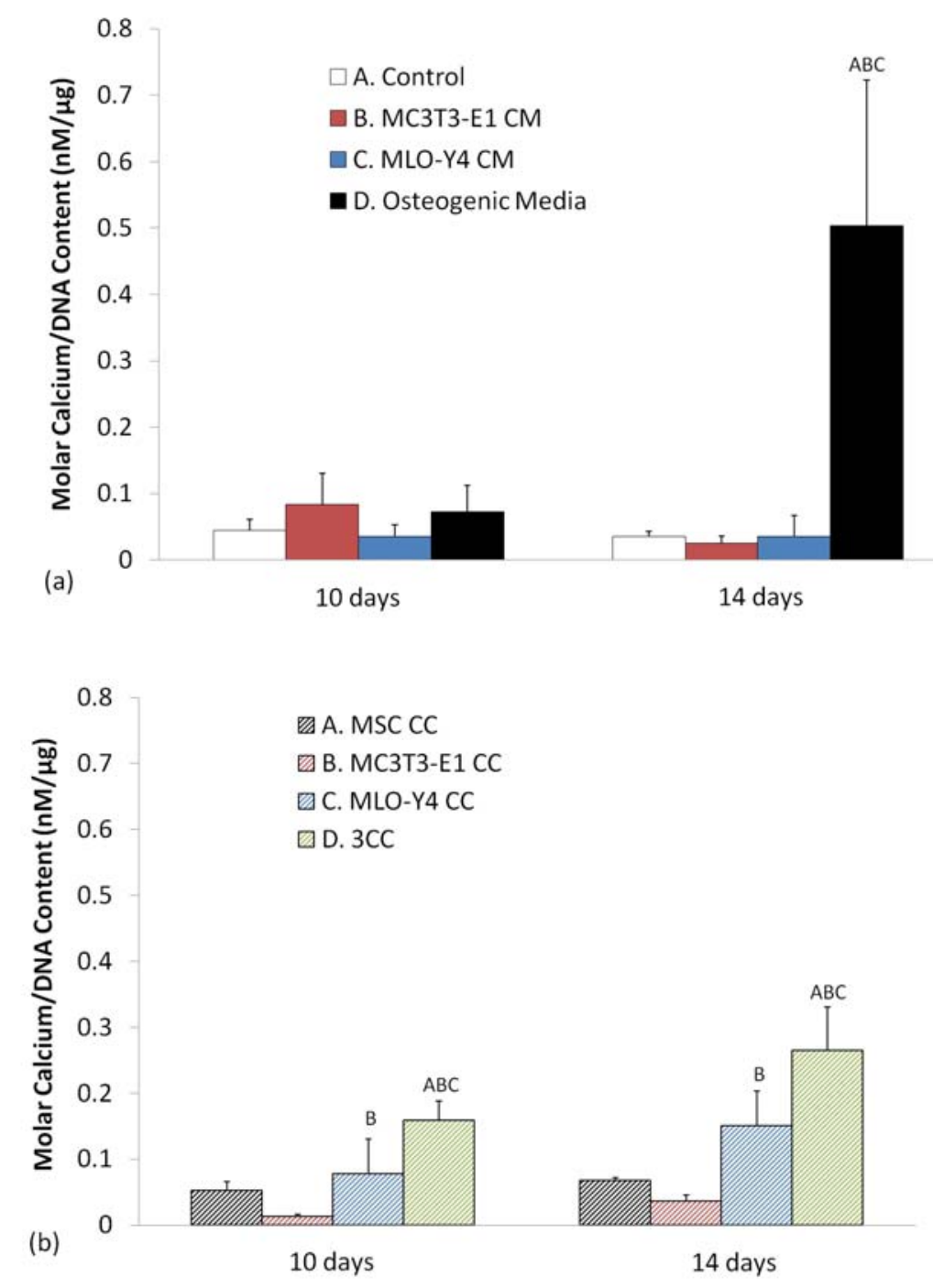

Fig. 6. Alizarin Red assay for calcium normalised to DNA content per well measured at 10 and $14 \mathrm{~d}$ for (a) MSCs exposed to conditioned media from MLO-Y4 cells (MLO-Y4 CM) and MC3T3-E1 cells (MC3T3-E1 CM). Also shown is the calcium analysis for MSCs grown in normal complete expansion media (Control) and MSCs grown in osteogenic media (Osteogenic Media). A, $p<0.05$ versus Control; B, $p<0.05$ versus MC3T3-E1 CM; C, $p<0.05$ versus MLO-Y4 CM; D, $p<0.05$ versus Osteogenic Media. (b) MSCs co-cultured with MLOY4 cells (MLO-Y4 CC), MC3T3-E1 cells (MC3T3-E1 CC), a three-layer co-culture with both MLO-Y4 and MC3T3-E1 cells (3CC), and MSCs co-cultured with MSCs (MSC CC). A, $p<0.05$ versus MSC CC; B, $p<0.05$ versus MC3T3-E1 CC; C, $p<0.05$ versus MLO-Y4 CC; D $p<0.05$ versus 3 CC. Error bars denote standard deviation $(n=4)$.

0.0020). Similarly, at $14 \mathrm{~d}$ the greatest calcium deposition was seen in the $3 \mathrm{CC}$ group, which was significantly greater than all other groups (MLO-Y4 CC $p=0.0084$, MC3T3-E1 $\mathrm{CC} p<0.0001$, and MSC CC $p=0.0001$ ) (Fig. 6b).

\section{Discussion}

The results of this study show that culturing MSCs in the presence of bone cells can direct MSCs down the osteoblast lineage in the absence of osteogenic media. Moreover, these studies suggest that osteocytes are more influential than osteoblasts in stimulating osteogenesis in MSCs. Specifically; key markers of osteogenic differentiation (intracellular ALP and calcium deposition) were greater at earlier time points when MSCs were cocultured with osteocytes rather than osteoblasts or MSCs. Most interestingly, it was found that when MSCs were exposed to factors from both osteoblasts and osteocytes simultaneously, the osteogenic effect was higher than exposure to either cell type alone. This data confirms that a functional relationship exists in the osteocyte-osteoblast network. This study gives an important insight into the natural cues for osteogenic differentiation within the stem niche in vivo.

Typically, in vitro osteogenic factors are introduced directly into the culture medium of MSCs to drive the MSCs toward osteogenic differentiation. However, 
it is unlikely that these same factors (ascorbic acid, dexamethasone and $\beta$-glycerol phosphate) are present within the natural environment in vivo. In previous studies where enhanced osteogenic differentiation of MSCs was reported, by either conditioned media or in a co-culture with bone cells, medium applied to MSC cultures was supplemented with osteogenic factors (Lu et al., 2011; Ilmer et al., 2009; Wang et al., 2007; Heino et al., 2004). In contrast, Csaki et al. (2009) found a high density co-culture of osteoblasts and MSCs to produce as strong an osteogenic response as a co-culture supplemented with osteogenic medium. It should be emphasised that in the present study no additional differentiation factors were used in the MSC media. These results indicate that bone cells themselves or bone tissue extracts could be used to regulate osteogenic differentiation of MSCs without addition of osteogenic factors, which could have important implications for tissue engineering applications. Moreover, these results provide an important insight into the regulatory cells that are capable of producing biochemical factors required to drive MSC differentiation in vivo.

Osteocytes are terminally differentiated osteoblasts (Parfitt, 1977; Bonewald, 2007), which form a cellular syncytium within an extensive lacunar-canalicular network, composed of long dendritic processes which in turn form functional gap junctions with other osteocytes and osteoblasts (Doty, 1981; Cheng et al., 2001; Alford et al., 2003). There is increasing evidence that osteocytes act as the mechanotransducers for bone (Skerry et al., 1989; Cowin et al., 1995; Tatsumi et al., 2007; Burra et al., 2010) and are also instrumental for regulating osteoclast formation from monocyte precursors (You et al., 2008). Here, it has been observed that osteocytes are capable of inducing a greater osteogenic response in MSCs than osteoblasts, both in terms of an increase in ALP activity in MSCs, which is a characteristic early marker of osteogenesis, and an increase in mineral production (a later osteogenic differentiation marker). These data further support the hypothesis that osteocytes are regulators of bone cell biology and remodelling.

That osteocytes are more influential than osteoblasts in stimulating osteogenesis in MSCs (Fig. 6b) is in agreement with previous studies (Heino et al., 2004; Hoey et al., 2011). In this study, we additionally show that this effect is observed in the absence of osteogenic factors. Moreover, we show that while the regulatory effect of osteoblasts is less than that of osteocytes, osteoblasts are also capable - given time - of stimulating osteogenesis in MSCs. This is in agreement with previous studies, which showed that osteoblasts elicited an osteogenic response in MSCs when direct contact was allowed (Csaki et al., 2009; Wang et al., 2007). It is not known whether direct cell-to-cell contact between MSCs and osteoblasts actually occurs in vivo. However, other studies have shown that MSCs cultured with conditioned media collected from osteoblasts increase expression of early osteogenic markers such as ALP and bone sialoprotein (BSP) (Ilmer et al., 2009). Moreover, studies where the two cell types are physically separated by a porous membrane have similarly shown that molecular signals from osteoblasts up-regulate osteogenic markers such as BSP, Cbfa2 (Runx2) and osteocalcin in
MSCs (Lu et al., 2011; Ilmer et al., 2009; Wang et al., 2007). However, these results are in contrast to those studies that suggest that only osteocytes are capable of stimulating osteogenesis (Heino et al., 2004; Hoey et al., 2011). Discrepancies between previous studies regarding the regulatory role of osteocytes and osteoblasts might be explained due the use of different cell types and species. Alternatively, these differences might reflect the stage of differentiation of the signalling osteoblasts, which according to our results will have an impact on the timing of the osteogenic differentiation of MSCs.

Our DNA results indicate that, in the presence of osteoblast factors, MSCs tend to proliferate initially and significantly more so than those cultured with osteocyte factors. Our findings reveal that MSCs exposed to osteoblast factors produce more ALP for the entire population. However, when we factor in the DNA content (as a measure of cell number) under these conditions, we find that MSCs exposed to osteoblast factors have lower levels of ALP production per cell. During osteogenic differentiation, cells undergo proliferation followed by differentiation (Aubin, 2001; Huang et al., 2007), and both events do not occur simultaneously. It must also be noted that during normal osteogenic differentiation there is an initial peak in ALP production followed by a subsequent decrease as the cells mature and lay down mineral (Aubin, 2001; Huang et al., 2007; Thibault et al., 2010). Our osteocyte groups do indeed demonstrate an initial increase in ALP production, but by $7 \mathrm{~d}$ this is lower than in those exposed to osteoblast factors, while only the MSCs exposed to factors secreted from osteocytes produce mineral at the time points examined here. As such, these data indicate that MSCs cultured in the presence of factors from osteoblasts initially undergo proliferation, whereas those cultured in the presence of factors from osteocytes initially differentiate. We propose that after osteoblasts themselves have differentiated into a more mature phenotype or osteocytes (Dallas and Bonewald, 2010), they are then able to encourage MSCs down the same osteogenic pathway, something that could be overlooked in short term studies.

The biochemical signals that osteocytes and osteoblasts release to encourage an osteogenic response in MSCs remain unknown. Growth factors, such as those from the TGF- $\beta$ family, are known to have stimulatory effects on bone formation (Linkhart et al., 1996; Rawadi et al., 2003). However, blocking TGF- $\beta 3$ in MLO-Y4 medium given to MSCs had no significant effect on proliferation or osteoblast differentiation (Heino et al., 2004). Bone morphogenetic proteins (BMPs), such as BMP-2, are growth factors also belonging to the TGF- $\beta$ family, which are well established osteogenic stimulation factors (Chen et al., 2004). Fibroblast growth factor-2 (FGF-2) has been proven to be effective at promoting the expression of osteogenic markers in MSCs (Gupta et al., 2010). As osteoblasts are known to express high levels of IGFs as they differentiate (into osteocytes), it is probable this family of factors play a role (Birnbaum et al., 1995). Cbfa1 (Runx2) and osteocalcin are expressed in higher quantities by osteocytes than osteoblasts (Mikuni-Takagaki et al., 1995; Ducy et al., 1997) and might be responsible for the quicker 
osteogenic response in MSCs exposed to osteocyte factors. Certain pro-osteogenic signalling molecules, such as nitric oxide (NO) and prostaglandin E2 (PGE2), are released in large amounts from mechanically stimulated osteocytes in vitro (Klein-Nulend et al., 1995a; Klein-Nulend et al., 1995b; Keila et al., 2001), but are also expressed in static conditions (McAllister et al., 2000; Cheng et al., 2001). These signals could provide the stimuli for MSC osteogenic differentiation in vivo.

The use of the cell lines MLO-Y4 and MC3T3-E1 is a possible limitation to this study. However, both cell lines have been shown to be excellent representatives of primary osteocytes and osteoblasts (Sudo et al., 1983; Kato et al., 1997; Quarles et al., 1992; Bonewald, 1999). Additionally, the use of serum was required to achieve osteogenic differentiation. It is possible that the results of the conditioned media experiments were influenced by serum from the osteocytes or osteoblasts. However, in our co-culture studies the cells received the same medium both above and below the membrane, and in these conditions the observed effects were more pronounced. Therefore, it is unlikely that the differences between osteoblasts and osteocytes observed here were due to differences in serum.

There is an interesting difference in results between the co-culture and conditioned media experiments, as demonstrated by the mineralisation results (Fig. 6). The higher mineralisation levels in co-culture $(p=0.0060$ for MLO-Y4 CC versus MLO-Y4 CM at $14 \mathrm{~d}$ ), compared to conditioned media, suggest that the physical presence of a directing cell type (osteoblast or osteocyte), which is continually producing biochemicals, enhances the osteogenic response seen in MSCs. Moreover, this is more representative of the in vivo situation rather that the conditioned media set-up, which provides only intermittent snapshots of the biochemicals being produced. Previous studies have not compared such set-ups. It is probable that osteocytes and osteoblasts communicate with MSCs exclusively through secreted factors due to their positioning within the bone and along the bone surface (Bonewald, 2007; Hauge et al., 2001). Secreted factors from osteocytes may enter the MSC niche directly, or through functional gap junctions between osteocytes and osteoblasts that have been demonstrated in vitro (Yellowley et al., 2000; Kamioka et al., 2007; Taylor et al., 2007). As osteocytes are positioned inside the lacunar-canalicular network of the bone matrix and osteoblasts are found lining the bone, these functional gap junctions are also established in vivo (Doty, 1981; Donahue, 2000). We propose that the indirect three-layer co-culture system is an appropriate model of the in vivo stem cell niche, wherein direct cell to cell contact between osteocytes and osteoblasts occurs, but undifferentiated MSCs do not directly contact either cell type (Bonewald, 2007; Hauge et al., 2001). Results here show that there is a synergistic effect between osteocytes and osteoblasts in biochemical signalling to MSCs.

Using the novel experimental design employed here, which incorporated both osteocytes and osteoblasts in a three-layer culture, it was shown that biochemical signals produced from this relationship enhanced MSC osteogenic differentiation, as seen by the greatest mineralisation compared to osteocyte and osteoblast co-culture alone
(Fig. 6b). These results further emphasise a synergistic relationship between osteocytes and osteoblasts and support the hypothesis that the syncytium of osteocytes and osteoblasts regulates bone formation by means of osteocytes actively communicating with inactive osteoblasts lining the calcified bone to recruit new osteoblasts from the marrow (Skerry et al., 1989; Cowin et al., 1991; Burger and Klein-Nulend, 1999).

\section{Conclusions}

In this study we report that osteogenic differentiation of MSCs, as characterised by ALP activity and mineralisation, was greater when MSCs were co-cultured with osteocytes rather than osteoblasts. These results suggest that osteocytes are more influential than osteoblasts in stimulating osteogenesis in MSCs. However, as osteoblasts were seen to promote a sharp rise in the cell number of MSCs at the initial stages of culture as well as a later increase in ALP activity, it is seen that they too stimulate osteogenesis in MSCs just at a slower rate. A novel three-layer co-culture system confirmed a synergistic relationship between osteocytes and osteoblasts in producing biochemical signals to stimulate the osteogenic differentiation of MSCs. This provides important insights into the mechanisms at work within the native stem cell niche to stimulate osteogenic differentiation. Additionally, all results were achieved in the absence of additional osteogenic factors in the media, outlining a possible role for the use of coculture or conditioned media methodologies for tissue engineering applications.

\section{Acknowledgements}

The MLO-Y4 cell line was received as a kind gift from Professor Lynda Bonewald (School of Dentistry, University of Missouri, Kansas City, MO). The authors would like to acknowledge funding from the Irish Research Council for Science, Engineering and Technology, under the EMBARK program (E. Birmingham) and the Science Foundation Ireland E.T.S. Walton program 07/W.I./B1806 (G.L. Niebur).

\section{References}

Alford AI, Jacobs CR, Donahue HJ (2003) Oscillating fluid flow regulates gap junction communication in osteocytic MLO-Y4 cells by an ERK1/2 MAP kinasedependent mechanism small star, filled. Bone 33: 64-70.

Aubin JE (2001) Regulation of osteoblast formation and function. Rev Endocr Metab Disord 2: 81-94.

Awad HA, Boivin GP, Dressler MR, Smith FNL, Young RG, Butler DL (2003) Repair of patellar tendon injuries using a cell-collagen composite. J Orthop Res 21: 420-431.

Birnbaum RS, Bowsher RR, Wiren KM (1995) Changes in IGF-I and -II expression and secretion during the proliferation and differentiation of normal rat osteoblasts. J Endocrinol 144: 251-259. 
Bonewald LF (1999) Establishment and characterization of an osteocyte-like cell line, MLOY4. J Bone Miner Metab 17: 61-65.

Bonewald LF (2007) Osteocytes as dynamic multifunctional cells. Ann NY Acad Sci 1116: 281-290.

Burger EH, Klein-Nulend J (1999) Mechanotransduction in bone - role of the lacunocanalicular network. FASEB J 13 Suppl: S101-112.

Burra S, Nicolella DP, Francis WL, Freitas CJ, Mueschke NJ, Poole K, Jiang JX (2010) Dendritic processes of osteocytes are mechanotransducers that induce the opening of hemichannels. Proc Natl Acad Sci USA 107: 13648-13653.

Butler DL, Gooch C, Kinneberg KRC, Boivin GP, Galloway MT, Nirmalanandhan VS, Shearn JT, Dyment NA, Juncosa-Melvin N (2010) The use of mesenchymal stem cells in collagen-based scaffolds for tissue-engineered repair of tendons. Nat Protoc 5: 849-863.

Chen D, Zhao M, Mundy GR (2004) Bone morphogenetic proteins. Growth Factors 22: 233-241.

Cheng B, Zhao S, Luo J, Sprague E, Bonewald LF, Jiang JX (2001) Expression of functional gap junctions and regulation by fluid flow in osteocyte-like MLO-Y4 cells. J Bone Miner Res 16: 249-259.

Cowin SC, Moss-Salentijn L, Moss ML (1991) Candidates for the mechanosensory system in bone. J Biomech Eng 113: 191-197.

Cowin SC, Weinbaum S, Zeng Y (1995) A case for bone canaliculi as the anatomical site of strain generated potentials. J Biomech 28: 1281-1297.

Csaki C, Matis U, Mobasheri A, Shakibaei M (2009) Co-culture of canine mesenchymal stem cells with primary bone-derived osteoblasts promotes osteogenic differentiation. Histochem Cell Biol 131: 251-266.

Dallas SL, Bonewald LF (2010) Dynamics of the transition from osteoblast to osteocyte. Ann NY Acad Sci 1192: $437-443$.

Dawson JI, Oreffo ROC (2008) Bridging the regeneration gap: stem cells, biomaterials and clinical translation in bone tissue engineering. Arch Biochem Biophys 473: 124-131.

Dominici M, Le Blanc K, Mueller I, Slaper-Cortenbach I, Marini F, Krause D, Deans R, Keating A, Prockop D, Horwitz E (2006) Minimal criteria for defining multipotent mesenchymal stromal cells. The International Society for Cellular Therapy position statement. Cytotherapy 8: 315 317.

Donahue H. (2000) Gap junctions and biophysical regulation of bone cell differentiation. Bone 26: 417-422.

Doty SB (1981) Morphological evidence of gap junctions between bone cells. Calcif Tissue Int 33: 509512.

Ducy P, Zhang R, Geoffroy V, Ridall AL, Karsenty G (1997) Osf2/Cbfa1: a transcriptional activator of osteoblast differentiation. Cell 89: 747-754.

Fuchs E, Tumbar T, Guasch G (2004) Socializing with the neighbors: stem cells and their niche. Cell 116: 769778.

Gu Y, Preston MR, El Haj AJ, Howl JD, Publicover SJ (2001) Three types of $\mathrm{K}(+)$ currents in murine osteocytelike cells (MLO-Y4). Bone 28: 29-37.
Gupta RR, Yoo DJ, Hebert C, Niger C, Stains JP (2010) Induction of an osteocyte-like phenotype by fibroblast growth factor-2. Biochem Biophys Res Commun 402: 258- 264.

Haleem AM, Singergy AAE, Sabry D, Atta HM, Rashed LA, Chu CR, El Shewy MT, Azzam A, Abdel Aziz MT (2010) The clinical use of human culture-expanded autologous bone marrow mesenchymal stem cells transplanted on platelet-rich fibrin glue in the treatment of articular cartilage defects: A pilot study and preliminary results. Cartilage 1: 253-261.

Hauge EM, Qvesel D, Eriksen EF, Mosekilde L, Melsen $\mathrm{F}$ (2001) Cancellous bone remodeling occurs in specialized compartments lined by cells expressing osteoblastic markers. J Bone Miner Res 16: 1575-1582.

Heino TJ, Hentunen TA, Väänänen HK (2004) Conditioned medium from osteocytes stimulates the proliferation of bone marrow mesenchymal stem cells and their differentiation into osteoblasts. Exp Cell Res 294: 458-468.

Hoemann CD, El-Gabalawy H, McKee MD (2009) In vitro osteogenesis assays: influence of the primary cell source on alkaline phosphatase activity and mineralization. Pathol Biol 57: 318-323.

Hoey DA, Kelly DJ, Jacobs CR (2011) A role for the primary cilium in paracrine signaling between mechanically stimulated osteocytes and mesenchymal stem cells. Biochem Biophys Res Commun 412: 182-187.

Huang Z, Nelson ER, Smith RL, Goodman SB (2007) The sequential expression profiles of growth factors from osteoprogenitors [correction of osteroprogenitors] to osteoblasts in vitro. Tissue Eng 13: 2311-2320.

Ilmer M, Karow M, Geissler C, Jochum M, Neth P (2009) Human osteoblast-derived factors induce early osteogenic markers in human mesenchymal stem cells. Tissue Eng A 15: 2397-2409.

Jaiswal N, Haynesworth SE, Caplan AI, Bruder SP (1997) Osteogenic differentiation of purified, cultureexpanded human mesenchymal stem cells in vitro. J. Cell Biochem 64: 295-312.

Kamioka H, Ishihara Y, Ris H, Murshid SA, Sugawara Y, Takano-Yamamoto T, Lim S-S (2007) Primary cultures of chick osteocytes retain functional gap junctions between osteocytes and between osteocytes and osteoblasts. Microsc Microanal 13: 108-117.

Kato Y, Windle JJ, Koop BA, Mundy GR, Bonewald LF (1997) Establishment of an osteocyte-like cell line, MLO-Y4. J Bone Miner Res 12: 2014-2023.

Keila S, Kelner A, Weinreb M (2001) Systemic prostaglandin E2 increases cancellous bone formation and mass in aging rats and stimulates their bone marrow osteogenic capacity in vivo and in vitro. J Endocrinol. 168: 131-139.

Klein-Nulend J, van der Plas A, Semeins CM, Ajubi NE, Frangos JA, Nijweide PJ, Burger EH (1995a) Sensitivity of osteocytes to biomechanical stress in vitro. FASEB J 9: 441-445.

Klein-Nulend J, Semeins CM, Ajubi NE, Nijweide PJ, Burger EH (1995b) Pulsating fluid flow increases nitric oxide (NO) synthesis by osteocytes but not periosteal 
fibroblasts - correlation with prostaglandin upregulation. Biochem Biophys Res Commun 217: 640-648.

Kuhn NZ, Tuan RS (2010) Regulation of stemness and stem cell niche of mesenchymal stem cells: implications in tumorigenesis and metastasis. J Cell Physiol 222: 268-277.

Li L, Xie T (2005) Stem cell niche: structure and function. Annu Rev Cell Dev Biol 21: 605-631.

Linkhart TA, Mohan S, Baylink DJ (1996) Growth factors for bone growth and repair: IGF, TGF[beta] and BMP. Bone 19: S1-S12.

Lu Z, Roohani I, Kwok PCL, Zreiqat H (2011) Osteoblasts on rod shaped hydroxyapatite nanoparticles incorporated PCL film provide an optimal osteogenic niche for stem cell differentiation. Tissue Eng A 17: 1651-1661.

Mackay AM, Beck SC, Murphy JM, Barry FP, Chichester CO, Pittenger MF (1998) Chondrogenic differentiation of cultured human mesenchymal stem cells from marrow. Tissue Eng 4: 415-428.

McAllister TN, Du T, Frangos JA (2000) Fluid shear stress stimulates prostaglandin and nitric oxide release in bone marrow-derived preosteoclast-like cells. Biochem Biophys Res Commun 270: 643-648.

Mikuni-Takagaki Y, Kakai Y, Satoyoshi M, Kawano E, Suzuki Y, Kawase T, Saito S (1995) Matrix mineralization and the differentiation of osteocyte-like cells in culture. $\mathrm{J}$ Bone Miner Res 10: 231-242.

Nørgaard R, Kassem M, Rattan SIS (2006) Heat shockinduced enhancement of osteoblastic differentiation of hTERT-immortalized mesenchymal stem cells. Ann NY Acad Sci 1067: 443-447.

Parfitt AM (1977) The cellular basis of bone turnover and bone loss: a rebuttal of the osteocytic resorption - bone flow theory. Clin Orthop Relat Res 236-247.

Peister A, Mellad JA, Larson BL, Hall BM, Gibson LF, Prockop DJ (2004) Adult stem cells from bone marrow (MSCs) isolated from different strains of inbred mice vary in surface epitopes, rates of proliferation, and differentiation potential. Blood 103: 1662- 1668.

Pittenger MF, Mackay AM, Beck SC, Jaiswal RK, Douglas R, Mosca JD, Moorman MA, Simonetti DW, Craig S, Marshak DR (1999) Multilineage potential of adult human mesenchymal stem cells. Science 284: 143-147.

Quarles LD, Yohay DA, Lever LW, Caton R, Wenstrup RJ (1992) Distinct proliferative and differentiated stages of murine MC3T3-E1 cells in culture: An in vitro model of osteoblast development. J Bone Miner Res 7: 683-692. Rawadi G, Vayssière B, Dunn F, Baron R, Roman-Roman S (2003) BMP-2 controls alkaline phosphatase expression and osteoblast mineralization by a Wnt autocrine loop. J Bone Miner Res 18: 1842-1853.

Schofield R (1978) The relationship between the spleen colony-forming cell and the haemopoietic stem cell. Blood Cells 4: 7-25.

Skerry TM, Bitensky L, Chayen J, Lanyon LE (1989) Early strain-related changes in enzyme activity in osteocytes following bone loading in vivo. J Bone Miner Res 4: 783-788.

Sudo H, Kodama HA, Amagai Y, Yamamoto S, Kasai $S$ (1983) In vitro differentiation and calcification in a new clonal osteogenic cell line derived from newborn mouse calvaria. J Cell Biol 96: 191-198.
Tatsumi S, Ishii K, Amizuka N, Li M, Kobayashi T, Kohno K, Ito M, Takeshita S, Ikeda K (2007) Targeted ablation of osteocytes induces osteoporosis with defective mechanotransduction. Cell Metab 5: 464-475.

Taylor AF, Saunders MM, Shingle DL, Cimbala JM, Zhou Z, Donahue HJ (2007) Mechanically stimulated osteocytes regulate osteoblastic activity via gap junctions. Am J Physiol, Cell Physiol 292: C545-552.

Thibault RA, Scott Baggett L, Mikos AG, Kasper FK (2010) Osteogenic differentiation of mesenchymal stem cells on pregenerated extracellular matrix scaffolds in the absence of osteogenic cell culture supplements. Tissue Eng A 16: 431-440.

Vater C, Kasten P, Stiehler M (2011) Culture media for the differentiation of mesenchymal stromal cells. Acta Biomater 7: 463-477.

Wang Y, Volloch V, Pindrus MA, Blasioli DJ, Chen J, Kaplan DL (2007) Murine osteoblasts regulate mesenchymal stem cells via WNT and cadherin pathways: mechanism depends on cell-cell contact mode. J Tissue Eng Regen Med 1: 39-50.

Yellowley CE, Li Z, Zhou Z, Jacobs CR, Donahue HJ (2000) Functional gap junctions between osteocytic and osteoblastic cells. J Bone Miner Res 15: 209-217.

You L, Temiyasathit S, Lee P, Kim CH, Tummala P, Yao W, Kingery W, Malone AM, Kwon RY, Jacobs CR (2008) Osteocytes as mechanosensors in the inhibition of bone resorption due to mechanical loading. Bone 42: 172-179.

\section{Discussion with Reviewers}

Reviewer II: The authors presented the difference in conditioned media versus defined osteogenic media, how can the authors rule out that it is not an effect of increased concentration of the same osteogenic factors seen in osteogenic media?

Authors: Osteogenic medium was only added to the MC3T3-E1 cells as a pre-treatment to encourage differentiation as this cell line is regarded as preosteoblastic requiring additional osteogenic factors (Sudo et al., 1983). This was necessary to establish the cell phenotype (i.e. osteoblast-like) prior to the beginning of the study (Sudo et al., 1983). Following this pre-treatment, MC3T3-E1 medium was changed back to standard osteoblast medium with no additional osteogenic factors. After $24 \mathrm{~h}$ this medium was then added to the MSCs for the conditioned media studies. Similarly, in co-culture studies the osteoblast-seeded insert was added to the MSCs after $24 \mathrm{~h}$ culture in standard medium in the absence of osteogenic factors. Only in the osteogenic media group (positive control) were any MSCs exposed directly to osteogenic factors.

It is believed here that the osteocytes and osteoblasts are releasing factors that encourage the osteogenic differentiation of MSCs. However, it is unlikely that these factors include chemicals such as ascorbic acid, dexamethasone and $\beta$-glycerol phosphate, which are the key constituents of osteogenic media, as the authors are unaware of any evidence that osteocytes or osteoblasts are capable of secreting such chemicals. It is likely that the 
factors that are released by the osteocytes and osteoblasts are targeting similar proteins to the osteogenic medium additives which are responsible for producing the collagen, BMPs and phosphate secretion required for bone formation (Jäger et al., 2008).

Reviewer II: Are the authors planning to investigate the nature of the factor responsible for osteocyte/ osteocyteosteoblast communication to MSCs?

Authors: The authors believe this is a very exciting area of study with many possible factors at work. An interesting molecule to focus on could be PGE2, which has been shown to have anabolic effects for cancellous bone formation in aged rats (Keila et al., 2001). The same study found that when PGE2 was applied to bone marrow MSCs in vitro there was an increase in the number of mineral nodules. Other studies have found PGE2 to have a profound anabolic effect in both in vitro studies on cells and animal models (Yoshida et al., 2002; Li et al., 2003; Kato et al., 2007). Due to the elevated expression of PGE2 by osteocytes, particularly under mechanical stimulation, it is a possible candidate molecule (Cherian et al., 2005). However, it has also been observed that added PGE2 had no additional osteogenic effect on osteoblasts that were co-cultured with flow-stimulated osteocytes (Taylor et al., 2007). Osteocytes are terminally differentiated osteoblasts and one of the characteristics of this differentiation is the expression of high quantities of osteocalcin (MikuniTakagaki et al., 1995). Osteocalcin is a protein crucial to the mineralisation of bone, and is promoted by the presence of Cbfa1(Runx2). Cbfa1 is regarded as essential for bone growth, and is an early marker for osteogenic differentiation (Ducy et al., 1997). That osteocytes express osteocalcin, and as such Cbfa1, in such high amounts suggests they can direct the osteogenic differentiation of MSCs. When ALP activity was analysed in this study, it was found that the MSCs exposed to osteoblast factors saw a high activity at later time points compared to MSCs exposed to osteocyte factors. This could be explained by the osteoblasts themselves differentiating and increasing the amount of later markers such as osteocalcin.

Reviewer II: Can the authors speculate on the role of gap junctions or hemichannels with regards to the described observations?

Authors: Hemichannels most likely play an important role in regulating the effects observed in this study, as they regulate the release of small molecules (e.g. PGE2) into the extracellular environment (Cherian et al., 2005). These unopposed connexins (hemichannels) are the likely method through which the osteocytes and osteoblasts are releasing signals to the MSCs. When two connexins come into contact, a gap junction is formed which allows for direct cell-to-cell contact. Functional gap junctions between osteocytes and osteoblasts are likely allowed to form in the three-layer co-culture through the porous membrane, based on the observations of gap junctions forming in co-culture on a membrane of the same pore size in a previous study (Taylor et al., 2007). As the three-layer culture was seen to have the greatest calcium deposition, it is hypothesised that the physical connections between the osteocytes and osteoblasts are amplifying the signalling to the MSCs. Moreover, this is representative of the in vivo situation where direct cell-to-cell contact between osteocytes and osteoblasts occurs, but undifferentiated MSCs found within the marrow most likely do not directly contact either cell type (Bonewald, 2007; Hauge et al., 2001). Gap junctions between osteocytes and osteoblasts have previously been shown to be essential in facilitating increased ALP activity by osteoblasts. Only the osteocytes were exposed to fluid shear, suggesting the gap junctions between the cells were essential for the increase in ALP activity (Taylor et al., 2007). In addition, the results presented in this study suggest that the functional network of osteocytes and osteoblasts are collectively recruiting undifferentiated MSCs to the osteogenic pathway. We propose that, in our two layer co-culture studies, hemichannels on osteocytes and osteoblasts are responsible for releasing signalling molecules into the media to induce osteogenic differentiation of MSCs (Burra et al., 2010), whereas in our 3 layer co-culture both gap junctions between osteocytes and osteoblasts and hemichannels play an important role in the observed osteogenic effect.

\section{Additional References}

Cherian PP, Siller-Jackson AJ, Gu S, Wang X, Bonewald LF, Sprague E, Jiang JX (2005) Mechanical strain opens connexin 43 hemichannels in osteocytes: a novel mechanism for the release of prostaglandin. Mol Biol Cell 16: 3100-3106.

Jäger M, Fischer J, Dohrn W, Li X, Ayers DC, Czibere A, Prall WC, Lensing-Höhn S, Krauspe R (2008) Dexamethasone modulates BMP-2 effects on mesenchymal stem cells in vitro. J Orthop Res 26: 1440-1448.

Kato N, Hasegawa U, Morimoto N, Saita Y, Nakashima K, Ezura Y, Kurosawa H, Akiyoshi K, Noda M (2007) Nanogel-based delivery system enhances PGE2 effects on bone formation. J Cell Biochem 101: 1063-1070.

Li M, Ke HZ, Qi H, Healy DR, Li Y, Crawford DT, Paralkar VM, Owen TA, Cameron KO, Lefker BA, Brown TA, Thompson DD (2003) A novel, non-prostanoid EP2 receptor-selective prostaglandin E2 agonist stimulates local bone formation and enhances fracture healing. J Bone Miner Res 18: 2033-2042.

Yoshida K, Oida H, Kobayashi T, Maruyama T, Tanaka M, Katayama T, Yamaguchi K, Segi E, Tsuboyama T, Matsushita M, Ito K, Ito Y, Sugimoto Y, Ushikubi F, Ohuchida S, Kondo K, Nakamura T, Narumiya S (2002) Stimulation of bone formation and prevention of bone loss by prostaglandin E EP4 receptor activation. Proc Natl Acad Sci USA 99: 4580-4585. 